\title{
Trust and experiences of NHS healthcare do not fully explain demographic disparities in coronavirus vaccination uptake in the UK
}

$26 / 5 / 2021$

Daniel Allington (Corresponding Author)

Department of Digital Humanities, King's College London, UK

Address: Department of Digital Humanities, King's College London, Strand, London WC2R 2LS

Email address: daniel.allington@kcl.ac.uk

Siobhan McAndrew

Sociology, Politics, and International Studies, University of Bristol, Bristol, UK

Bobby Duffy

Policy Institute, King's College London, London, UK

Vivienne Moxham-Hall

Policy Institute, King’s College London, London, UK

Word count: 4269 


\section{Trust and experiences of NHS healthcare do not fully explain demographic disparities in coronavirus vaccination uptake in the UK}

Daniel Allington, Siobhan McAndrew, Bobby Duffy, and Vivienne Moxham-Hall $26 / 5 / 2021$

\section{Abstract}

\section{Objectives}

To test whether demographic variation in vaccine hesitancy can be explained by differences in trust and healthcare experiences.

\section{Design}

Cross-sectional study.

\section{Setting}

Data collected online by Ipsos MORI in April 2021.

\section{Participants}

Data collected from 4885 UK-resident adults, of whom 3223 had received the invitation to be vaccinated against the novel coronavirus. The remainder were excluded. 1629 included participants identified as female and 1594 as male. 234 identified as belonging to other than white ethnic groups while 2967 identified as belonging to white ethnic groups.

\section{Primary and secondary outcome measures}

Uptake of coronavirus vaccination.

\section{Results}

Membership of an other than white ethnic group (AOR $=0.53,95 \% \mathrm{CI}[0.35,0.84], \mathrm{p}=.005$ ) and age (AOR $=1.61,95 \%$ CI $[1.39,1.87], \mathrm{p}<.001$ for a 1 SD change from the mean) were the only statistically significant demographic predictors of vaccine uptake. After controls for NHS healthcare experiences and trust in government, scientists, and medical professionals, the effect associated with membership of an other than white ethnic group appears more marginal (AOR $=0.61,95 \%$ CI $[0.38,1.01], \mathrm{p}=.046$ ), while the effect associated with age remains virtually unchanged. Exploratory analysis suggests that NHS healthcare 
experiences mediate 24\% (95\% CI [8\%, 100\%], $\mathrm{p}=.024)$ of the association between ethnicity and uptake, while trust mediates $94 \%$ (95\% CI [56\%, 100\%], p .001) of the association between NHS healthcare experiences and uptake.

\section{Conclusions}

Members of other than white ethnic groups report inferior NHS healthcare experiences, which may explain the lower trust that they report in government, scientists, and medical professionals. However, this does not fully explain the ethnic gap in coronavirus vaccination uptake.

\section{Trial registration}

The data collection and confirmatory analysis were pre-registered with the Center for Open Science (osf.io/56txk).

\section{Article summary}

\section{Strengths and limitations of this study}

- The sample was large, providing high statistical power, and was designed for representativeness of the UK adult population

- Standard measures of trust and healthcare experiences were used, providing confidence that these variables have been measured robustly

- However, it was not possible to obtain a probability sample, and there was limited representation within the sample both of people who did not take up the invitation to be vaccinated and of members of other than white ethnic groups

\section{Introduction}

The problem of vaccine hesitancy, defined as 'delay in acceptance or refusal of vaccination despite availability of vaccination services’ [1], has been recognised as a major global health problem for several years [2,3]. However, it has gained particular importance during the COVID-19 public health emergency: early in the pandemic, it was estimated that as much as $82 \%$ of a population might need vaccination or natural immunity against the disease before herd immunity could be reached [4], but one survey indicated that less than $72 \%$ of the British population might be willing to accept vaccination against SARS-CoV-2, the novel coronavirus which causes COVID-19 [5]. Multiple surveys found demographic disparities in British respondents' expressed likelihood of vaccine uptake, with younger, less educated respondents, women, members of lower income households, and members of other than white ethnic groups all tending to express a lower probability of accepting the offer of vaccination [6-8]. The finding with regard to ethnicity has been of particular concern, given higher fatality rates for COVID-19 among members of minority ethnic groups [9]. Actual uptake has been far higher than these surveys would have predicted, with one longitudinal study finding that 99\% of those who had stated in December 2020 that they would accept vaccination, and 86\% of those who had stated at the same point that they would not or were unsure, stated in February 2021 either that they would accept vaccination or that they had already done so [10]. However, despite this encouraging trend, demographic disparities appeared to persist [11], albeit at a reduced level, with youth and membership 
of other than white ethnic groups now being the strongest predictors of coronavirus vaccine hesitancy in the UK [10], such that by 13 January 2021, 42.5\% of white people aged over 80 but not resident in care homes, but only $20.5 \%$ of black people in the same category, had been vaccinated [12]. The potential health consequences of incomplete vaccination coverage were recently highlighted by the official claim that the majority of people hospitalised as a result of a COVID-19 outbreak in the English town of Bolton were unvaccinated yet eligible to be vaccinated [13].

The possibility that lower trust in government, scientists, and the medical system might lead to reduced coronavirus vaccine uptake among members of minority ethnic communities had already been noted before survey data of this nature had been collected [14]. Indeed, many studies have found a relationship between hesitancy with regard to other vaccinations and mistrust in medicine and other relevant institutions, both on an individual level [15-18] and on a national level [19]. Evidence now exists to suggest that coronavirus vaccine hesitancy among African Americans may be driven by mistrust in the medical establishment and by perceptions of racism in the political system [20]. Moreover, some experts have attributed heightened levels both of coronavirus misinformation and of coronavirus vaccine hesitancy among members of some minority ethnic communities in the UK to mistrust which they argue is in turn driven by experiences of racism, discrimination, and exclusion [21]. This could be related to a UK study's finding that trust in scientists, medical professionals, and (to a much lesser extent) government is predictive of coronavirus vaccine hesitancy [8]. The same study found that ethnic disparities were reduced but did not disappear following controls for trust in these institutions, which may indicate the importance of other drivers. As members of minority ethnic groups report lower patient satisfaction and worse healthcare experiences in the UK [22], and, on an international level, appear to suffer from 'higher incidences of healthcare associated infections, dosing errors, [adverse drug events], and complications resulting from their care' [23], it would seem plausible that healthcare experiences (which were not assessed in the aforementioned study) may act as a driver of ethnic disparities in coronavirus vaccine uptake, alongside strategic and situational trust. Moreover, it would appear plausible that the range of mechanisms which can be assumed to generate the forms of exclusion associated with racism might also be associated with decreased trust, more negative experiences of healthcare, and higher rates of vaccine hesitancy among other groups, including women, young people, less highly educated people, and members of low income households. In this context, it is important to note that a separate UK study found that '[s]ocio-demographics do not explain vaccine hesitancy to any helpful degree' and that the major predictors of coronavirus vaccine hesitancy are 'excessive mistrust' and a lack of 'positive healthcare experiences' [5]. These findings are startling, and urgently require interrogation through replication.

This article therefore reports on a pre-registered study designed in order to test for demographic predictors of coronavirus vaccination uptake in the UK, both before and after controls for healthcare experiences and for trust in the principal institutions associated with the UK's coronavirus vaccination programme, i.e. government, scientists, and medical professionals [24]. As the UK's vaccination programme is delivered by National Health Service (NHS) bodies in England, Scotland, Wales, and Northern Ireland, healthcare experience items focused specifically on experiences of NHS healthcare. In order to provide the most robust test possible, established instruments have been used to measure both trust and healthcare experiences, rather than the novel measures employed in the aforementioned study [5]. 


\section{Hypotheses}

Given the above observations regarding published findings of existing research, the following expectations were formed:

H1. Coronavirus vaccination uptake will be lower amongst female respondents

H2. Coronavirus vaccination uptake will be lower amongst younger respondents

H3. Coronavirus vaccination uptake will be lower amongst respondents of other than white ethnic groups

H4. Coronavirus vaccination uptake will be lower amongst less educated respondents

H5. Coronavirus vaccination uptake will be lower amongst respondents from lower-income households

H6. Coronavirus vaccination uptake will be positively associated with trust in government, medical professionals, and scientists

H7. Coronavirus vaccination uptake will be positively associated with positive experiences of healthcare

\section{Methods}

\subsection{Study design}

Trust was measured using Wellcome Global Health Monitor items Q11B, Q11E, Q14A, and Q15a [25]. Experiences of healthcare were measured using items adapted from the Scottish Government Inpatient Experience Survey [26]. Responses to individual questions were numerically recoded and averaged to provide a single measure of trust and a single measure of experience of healthcare. To facilitate analysis of individual trust and healthcare items, responses to these items were separately dichotomised for use in additional partial models, with the top two levels recoded as true and others recoded as false.

Education was operationalised as highest qualification received, which was dichotomised in order to distinguish degree-level (and equivalent) qualifications (including undergraduate and postgraduate degrees) from lower levels of qualification, including lack of all formal qualifications. Ethnic group was also dichotomised through coding as white or other than white, and household income was measured on an ordinal scale and dichotomised through coding as below median (for the sample) and median or above. In order to accommodate respondents who do not identify as male or female, gender was operationalised as a categorical variable with only two values: female and other than female. However, all participants included in the survey identified either as male or as female.

The dependent variable is a dichotomous variable representing the condition of having been vaccinated against the novel coronavirus (in the event that the respondent has been invited to be vaccinated) or of being about to attend an appointment to be vaccinated (in the event that a respondent was surveyed immediately after being invited to be vaccinated, and for that reason has not been vaccinated yet).

In calculating indices, missing values were ignored. Cases with missing data in relation to specific variables were excluded from all analyses featuring those variables.

\subsection{Data collection}

Although there is no longitudinal aspect to the study reported here, the data collection formed part of a longitudinal study in which each wave involves collection both from recontactees and from new participants. 
Respondents were sampled at random from an Ipsos MORI recruited panel, with pre-stratification in order to produce representativeness of the UK adult population on age, gender, geographical region, and working status. Where representativeness was not achieved, additional respondents were obtained by Ipsos MORI on a quota basis from panels maintained by other data providers. Data were collected from respondents who have not been offered vaccination against the novel coronavirus, but data on those respondents were excluded from this particular study, as they could not be used to test hypotheses about vaccine uptake.

Data collection was carried out online by Ipsos MORI. Fieldwork was launched on 01 April 2021 and completed on 16 April 2021. Informed consent was obtained from all participants. Demographic weights were calculated post-collection by Ipsos MORI on the basis of education and geographical region and of gender interlocked with age, NRS social grade, and working status. Weights were calculated before exclusion of cases where respondents had not yet been invited to receive coronavirus vaccination. Data were collected from a total of 4885 respondents, of whom 1662 were excluded due to not having received the invitation to be vaccinated. Numbers of included cases with missing values for each variable are listed in Table $\underline{3.1}$.

Table 3.1: Included cases with missing values

\begin{tabular}{|c|c|c|c|c|c|c|c|c|}
\hline & Age & Gender & Degree & Other than white & Low income & Trust & NHS experiences & Uptake \\
\hline Missing & 0 & 0 & 0 & 22 & 305 & 8 & 124 & 46 \\
\hline
\end{tabular}

\subsection{Statistical analysis}

The sample was treated as equivalent to a random sample for analytic purposes. Hypotheses 1-7 were tested through creation of binomial logit models with vaccine acceptance as the dependent variable. Of most relevant interest are a partial model using only demographic predictors, and the full model featuring demographic predictors and aggregate measures of trust and of experiences of NHS healthcare. Both of these models were pre-registered. To further probe the findings of the confirmatory analysis (see below), two further partial models were created without having been pre-registered. These included one featuring demographic predictors and aggregate measures of trust only and one featuring demographic predictors and aggregate measures of NHS healthcare only. In order to study the predictiveness of individual items, two further pre-registered models were created, one featuring demographic predictors and dichotomised responses to trust items as predictors, and the other featuring demographic variables and dichotomised responses to healthcare experience items as predictors. To further understand the unexpected findings of the confirmatory analyses, mediation analyses were conducted on an exploratory basis using non-parametric bootstrapping with 10000 repetitions. Coefficients for all confirmatory and exploratory analyses are reported both as estimates and as 95\% confidence intervals.

\subsection{Power analysis}

Power analysis confirms that the sample was sufficiently large to detect even very small effects, at least in the pre-registered models. Given a sample size of 2773 (i.e. the number of observations which were sufficiently complete to be fitted in the full model) and a cut-off of $\mathrm{p}<.050$ (two-tailed), an association so weak as to reduce the probability of vaccine uptake only from $95 \%$ to $91 \%$ across the entire range of a predictor variable could be detected with approximately $98 \%$ power. 


\subsection{Patient and public involvement}

No patient involved.

\section{Results}

\subsection{Descriptive statistics and internal reliability}

Table 4.1: Descriptive statistics: demographic variables

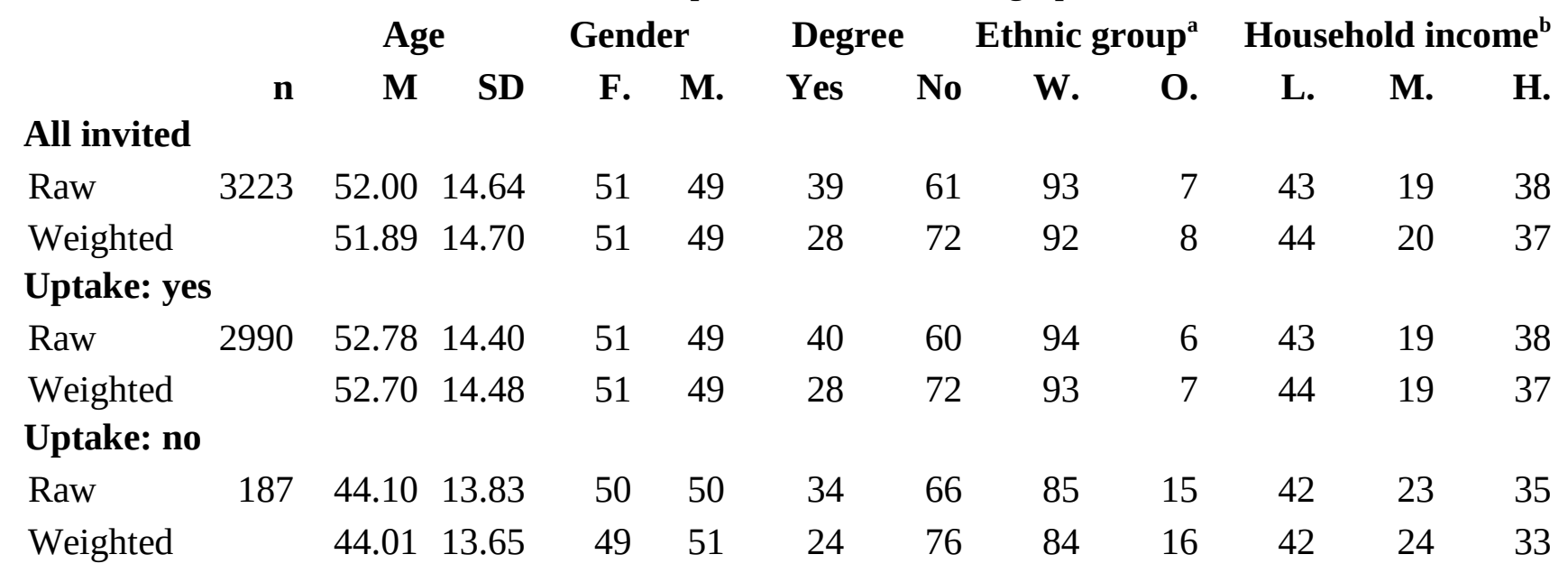

a White ethnic groups comprise white British, English, Welsh, Scottish, Northern Irish, Irish, Gypsy or Irish Traveller, and any other white background; other than white ethnic groups comprise all other ethnic categories used in the 2011 UK census, including mixed ethnicities

b Low household income $=£ 24,999$ or less; median household income (for included participants) $=£ 25,000$ $£ 34,999$; high household income $=£ 35,000$ or more

Cronbach's alpha and Guttman's lambda 6 were used to assess internal reliability of indices. The reliability of items used to measure experiences of NHS healthcare was assessed as $\alpha=.88, \lambda_{6}=.87$, which indicates a high level of internal reliability. The reliability of items used to measure trust was assessed as $\alpha=.69$, $\lambda_{6}$ $=.66$, which would be a suboptimal level of internal reliability for a conventional Likert scale but likely reflects real differences in levels of trust for different institutions (especially given that government trust may be acting at least partly as a proxy for political preference). 
Table 4.2: Descriptive statistics: indices

\section{NHS exp. Trust}

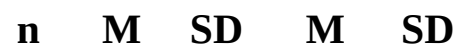

\begin{tabular}{lrrrrr} 
All invited & & & & & \\
\hline Raw & 3223 & 4.06 & 0.71 & 3.09 & 0.52 \\
Weighted & & 4.05 & 0.71 & 3.08 & 0.53 \\
Uptake: yes & & & & & \\
\hline Raw & 2990 & 4.08 & 0.69 & 3.12 & 0.48 \\
Weighted & & 4.08 & 0.69 & 3.12 & 0.48 \\
Uptake: no & & & & & \\
\hline Raw & 187 & 3.70 & 0.92 & 2.64 & 0.77 \\
Weighted & & 3.69 & 0.94 & 2.64 & 0.78
\end{tabular}

Table 4.3: Key variables: bivariate correlations

$\begin{array}{lrrrrrrrr}\text { 1. Age } & & -.01 & -.27 & .11 & -.09 & .10 & .06 & .14 \\ \text { 2. Female } & -.01 & & -.04 & .05 & .05 & .01 & .05 & .00 \\ \text { 3. Other than white } & -.27 & -.04 & & -.01 & .03 & -.13 & -.08 & -.08 \\ \text { 4. Low income } & .11 & .05 & -.01 & & -.21 & -.03 & -.06 & .00 \\ \text { 5. Degree } & -.09 & .05 & .03 & -.21 & & .04 & .04 & .03 \\ \text { 6. NHS experiences } & .10 & .01 & -.13 & -.03 & .04 & & .47 & .13 \\ \text { 7. Trust } & .06 & .05 & -.08 & -.06 & .04 & .47 & & .22 \\ \text { 8. Uptake } & .14 & .00 & -.08 & .00 & .03 & .13 & .22 & \end{array}$

Table 4.1 shows weighted and unweighted demographic descriptive statistics, first for the whole sample, and then for respondents who did and did not accept the invitation to be vaccinated against the novel coronavirus. Although respondents from households with income at or above the median for the sample were treated as a single group for modelling purposes, they are disaggregated here. Comparison with official statistics suggests slight representation of white ethnic groups, which accounted for $86 \%$ of the population at the 2011 census, but the sample was biased towards older members of the population as vaccination was offered in descending order of age, and other than white ethnic groups have a younger age profile in the UK [27].

The mean age for respondents who had been invited to be vaccinated yet not take up the invitation is just under nine years younger than the mean age for respondents who had been vaccinated. Percentages of male and female and respondents in each group are identical. Percentages with low (below median) household incomes for the sample are effectively identical, but the weighted percentage of respondents with high (above median) household incomes for the sample is 4 points lower among those who did not accept the invitation to be vaccinated (i.e. it was about 0.9 times as high). This suggests that the effect of income may be masked in the confirmatory analysis presented in this article, as dichotomisation by separating low income households from median and high income households was a pre-registered data transformation: a point to which we shall return below. The weighted percentage of respondents educated to degree level was 4 points lower among those who did not accept the invitation to be vaccinated (i.e. it was about 0.9 times as high). The weighted percentage of respondents belonging to other than white ethnic groups was 9 points higher among those who 
did not accept the invitation to be vaccinated (i.e. it was about 2.3 times as high). This is not a large difference: by the time of data collection, $94 \%$ and $87 \%$ of invited members of other than white ethnic groups within the sample who had received the invitation to be vaccinated had taken it up, which implies that the great majority of members of all ethnic groups had done so. However, it is consistent with the observation, discussed above, that uptake rates have been lower amongst members of other than white ethnic groups.

Table 4.2 shows weighted and unweighted indices of trust and NHS healthcare experiences, first for the whole sample, and then for respondents who did and did not accept the invitation to be vaccinated against the novel coronavirus. Mean scores for members of the latter group were lower on both indices. Table $\underline{4.3}$ shows unweighted product-moment correlations between pairs of variables. There was a strong correlation between indices of trust and NHS healthcare experiences $(r=.47)$, but NHS healthcare experiences were less strongly correlated with vaccine uptake $(r=.13)$ than trust was $(r=.22)$, while trust was less strongly correlated with membership of an other than white ethnic group $(r=-.08)$ than NHS healthcare experiences were $(r=-.13)$. Age was negatively correlated with membership of an other than white ethnic group $(r=-.27)$ and with degree level education ( $r=-.09$ ), but positively correlated with membership of a low income household ( $\mathrm{r}$ $=.11)$ and with vaccination uptake $(r=.14)$. 
Table 4.4: Partial models and full model

AOR $2.5 \% \quad 97.5 \% \quad$ p

Demographics only

\begin{tabular}{lllll}
\hline Age & 1.61 & 1.39 & 1.87 & $<.001$
\end{tabular}

$\begin{array}{lllll}\text { Female } & 0.96 & 0.71 & 1.30 & .808\end{array}$

$\begin{array}{lllll}\text { Other than white } & 0.53 & 0.35 & 0.84 & .005\end{array}$

$\begin{array}{lllll}\text { Low income } & 1.02 & 0.75 & 1.40 & .883\end{array}$

$\begin{array}{lllll}\text { Degree } & 1.30 & 0.92 & 1.87 & .140\end{array}$

NHS experiences

\begin{tabular}{lllll}
\hline Age & 1.56 & 1.34 & 1.83 & $<.001$
\end{tabular}

$\begin{array}{lllll}\text { Female } & 0.98 & 0.71 & 1.34 & .882\end{array}$

$\begin{array}{lllll}\text { Other than white } & 0.65 & 0.41 & 1.04 & .064\end{array}$

$\begin{array}{lllll}\text { Low income } & 1.09 & 0.79 & 1.51 & .591\end{array}$

$\begin{array}{lllll}\text { Degree } & 1.29 & 0.91 & 1.87 & .167\end{array}$

$\begin{array}{lllll}\text { NHS experiences } & 1.46 & 1.27 & 1.67 & <.001\end{array}$

Trust

\begin{tabular}{llllr}
\hline Age & 1.57 & 1.34 & 1.85 & $<.001$ \\
Female & 0.89 & 0.65 & 1.23 & .490 \\
Other than white & 0.61 & 0.39 & 0.98 & .037 \\
Low income & 1.12 & 0.81 & 1.56 & .491 \\
Degree & 1.18 & 0.82 & 1.71 & .380 \\
Trust & 2.06 & 1.80 & 2.36 & $<.001$
\end{tabular}

Full model

\begin{tabular}{llllr}
\hline Age & 1.57 & 1.33 & 1.86 & $<.001$ \\
Female & 0.92 & 0.66 & 1.28 & .615 \\
Other than white & 0.61 & 0.38 & 1.01 & .046 \\
Low income & 1.16 & 0.83 & 1.63 & .379 \\
Degree & 1.22 & 0.84 & 1.80 & .297 \\
NHS experiences & 1.02 & 0.86 & 1.20 & .820 \\
Trust & 2.02 & 1.72 & 2.37 & $<.001$
\end{tabular}


Table 4.5: Individual trust and NHS healthcare experience items (dichotomised)

AOR $2.5 \% \quad 97.5 \% \quad$ p

NHS experiences

\begin{tabular}{lrrrr}
\hline Age & 1.51 & 1.30 & 1.77 & $<.001$ \\
Female & 0.91 & 0.67 & 1.24 & .556 \\
Other than white & 0.64 & 0.41 & 1.01 & .049 \\
Low income & 1.03 & 0.75 & 1.41 & .848 \\
Degree & 1.24 & 0.88 & 1.79 & .226 \\
Respect & 0.99 & 0.61 & 1.59 & .967 \\
Care & 1.17 & 0.72 & 1.89 & .513 \\
Needs & 1.45 & 0.96 & 2.18 & .077 \\
Trust staff & 1.62 & 1.01 & 2.58 & .045 \\
Understand & 1.37 & 0.89 & 2.11 & .148 \\
Spiritual & 0.76 & 0.54 & 1.07 & .113 \\
Trust & & & & \\
\hline Age & 1.52 & 1.30 & 1.78 & $<.001$ \\
Female & 0.91 & 0.67 & 1.24 & .552 \\
Other than white & 0.68 & 0.43 & 1.09 & .097 \\
Low income & 1.12 & 0.82 & 1.54 & .487 \\
Degree & 1.20 & 0.84 & 1.72 & .322 \\
Trust UK gov. & 1.48 & 1.07 & 2.07 & .021 \\
Trust sci. uni. & 1.77 & 1.12 & 2.77 & .013 \\
Trust sci. corp. & 1.94 & 1.34 & 2.78 & $<.001$ \\
Trust medics & 2.81 & 1.74 & 4.49 & $<.001$
\end{tabular}

\subsection{Confirmatory analysis}

Table 4.4 presents adjusted odds ratios (with 95\% confidence intervals) for dichotomous variables (female gender, membership of an other than white ethnic group, membership of a low income household, and education to undergraduate or postgraduate degree level) and for increases of 1 SD in numeric variables (i.e. age and the indices for trust and NHS healthcare experiences). Age (AOR = 1.61, 95\% CI [1.39, 1.87], p $<.001$ ) and membership of an other than white ethnic group (AOR $=0.53,95 \%$ CI [0.35, 0.84], $\mathrm{p}=.005$ ) were the only statistically significant predictors in the purely demographic model. Thus, the null hypothesis can be rejected with regard to $\mathrm{H} 2$ and $\mathrm{H} 3$, but not with regard to H1, H4, and $\mathrm{H} 5$.

Controlling for indices of trust and of NHS healthcare experiences diminishes the effect associated with membership of an other than white ethnic group from AOR $=0.53,95 \% \mathrm{CI}[0.35,0.84], \mathrm{p}=.005$ to AOR = $0.61,95 \%$ CI $[0.38,1.01], \mathrm{p}=.046$. This suggests that some of the effect associated with ethnicity may be mediated by one or both of those additional variables. However, the same cannot be said for age. Indeed, the null hypothesis can only be rejected for $\mathrm{H} 3$ with regard to this model at $\mathrm{p}<.050$, but the coefficient and $\mathrm{p}$ value relevant to $\mathrm{H} 2$ are virtually unchanged relative to the demographic-only model. Moreover, while there was a significant effect associated with trust in the full model (AOR = 2.02, 95\% CI [1.72, 2.37], p <.001), there was no effect associated with NHS healthcare experiences (AOR = 1.02, 95\% CI [0.86, 1.2], p = .820). 
Thus, the null hypothesis can be rejected with regard to H6, but not with regard to H7.

This finding would appear to be in contradiction of the claim that vaccine hesitancy was largely explained by trust alongside healthcare experiences, and that demographic predictors have little importance. Partial models excluding trust or healthcare experiences are also presented in Table 4.4. As the effect associated with NHS healthcare experiences in the partial model excluding trust is very highly significant (AOR $=1.46,95 \%$ CI [1.27, 1.67], $\mathrm{p}<.001$ ), albeit lower than the effect associated with trust in the partial model excluding NHS healthcare experiences $(\mathrm{AOR}=2.06,95 \% \mathrm{CI}[1.8,2.36], \mathrm{p}<.001, \mathrm{AOR}=2.06,95 \% \mathrm{CI}[1.8,2.36], \mathrm{p}$ $<.001$ ), it seems plausible that the effect of NHS healthcare experiences on uptake may be mediated by trust: a possibility to which we shall return below.

Table 4.5 presents adjusted odds ratios (with 95\% confidence intervals) for increases of 1 SD in age and for dichotomous variables, including dichotomised trust and NHS healthcare items as individual predictors. Examination of coefficients associated with those items facilitates assessment of their individual predictive contribution. All effects associated with trust items are statistically significant, especially those associated with trust in medical professionals (AOR $=2.81,95 \%$ CI $[1.74,4.49], \mathrm{p}<.001)$ and with scientists working in the private sector $(\mathrm{AOR}=1.94,95 \% \mathrm{CI}[1.34,2.78], \mathrm{p}<.001)$. Thus, high trust in any of the institutions mentioned was associated with increased likelihood of uptake, even after controlling for level of trust in all the others, plus demographic variables. Interestingly, the only significant effect associated with an individual NHS healthcare experience item after controlling for all the other items and for demographic variables was that associated with having felt able to trust the staff (AOR $=1.62,95 \%$ CI $[1.01,2.58], \mathrm{p}=.045$ ). In relation to these models, the null hypothesis is still rejected with regard to $\mathrm{H6}$, and may be partially rejected with regard to $\mathrm{H} 7$.

\subsection{Exploratory analysis}

The above findings were suggestive of theoretically plausible mediated associations, i.e. that experiences of NHS healthcare might be indirectly associated with uptake via trust, that ethnicity might be indirectly associated with trust via experiences of NHS healthcare, and that ethnicity might therefore be indirectly associated with uptake via both of the aforementioned. For this reason, additional mediation analyses were carried out on an exploratory basis (i.e. without having been pre-registered). All of these analyses involved the same demographic controls used in the confirmatory analyses.

There were three findings. Firstly, the relationship between membership of an other than white ethnic group and trust appears to be $100 \%(95 \%$ CI [48\%, 100\%], p = .040) mediated by NHS healthcare experiences. 1 Secondly, the relationship between NHS healthcare experiences and uptake appears to be 94\% (95\% CI [56\%, 100\%], p .001) mediated via trust. Thirdly, the association between membership of an other than white ethnic group and uptake appears to be $24 \%(95 \%$ CI [8\%, 100\%], p = .024) mediated via NHS healthcare experiences (trust was not included in this model, given the second finding). This mediating effect may plausibly be taken to account for the reduction in the effect associated with ethnicity between the demographic-only model and the full confirmatory model.

While these findings are exploratory rather than confirmatory, they add weight to the above interpretation of the confirmatory findings. On the one hand, they suggest that positive experiences of NHS healthcare may be associated with higher vaccine uptake largely for the reason that they have a positive relationship with trust in relevant institutions, and that membership of an other than white ethnic group is associated with lower trust in those same institutions for the reason that experiences of NHS healthcare are less positive among 
members of such groups. On the other hand, these findings also suggest that those intermediary relationships can only account for some of the association between ethnicity and vaccine uptake. Most of that association remains unexplained, as does the association between age and uptake.

\section{Discussion}

The finding that female gender, below degree-level education, and below median income do not predict uptake contradicts several studies which measured vaccine hesitancy before the UK vaccination campaign had got underway $[6-8,10]$. This discrepancy may be interpreted in terms of an attitude change potentially attributable to public health communication, word of mouth discussion of coronavirus vaccination itself, or many other sources (although it is also possible that members of certain groups simply under-estimated their own likelihood of accepting vaccination).

The finding that age and ethnicity do, however, predict uptake even after controls for trust and NHS healthcare experiences is in contradiction of the earlier finding that vaccine hesitancy is largely explained by mistrust and poor experiences of healthcare, to such an extent that demographic variables are not predictive once these variables are controlled for [5]. Non-replication of that finding might be accounted for by the timeframe (data collected after the vaccination programme had begun), outcome measure (actual uptake rather than expected uptake), predictor measures (those used here both for trust and for healthcare experiences were more standard), or analytic approach (pre-registered models rather than exploratory modelling). Age is here found to remain a powerful predictor of uptake even after those controls, and ethnicity also predicts uptake, albeit that this effect appears weaker after the same controls. This reduction in strength of association appears to be the result of mediation by NHS healthcare experiences, via trust. However, the mediation effect is small, appearing to account for about a quarter of the variation in uptake which is associated with ethnicity. To summarise, this study finds evidence that uptake of coronavirus vaccination is lower among members of other than white ethnic groups, that NHS healthcare experiences have a relationship with vaccine uptake that is mediated by trust, and that the lower trust in medics, scientists, and government among members of other than white ethnic groups is mediated by their worse experiences of NHS healthcare. However, it does not find evidence that worse healthcare experiences or lower trust can explain more than a small part of the ethnicity gap in coronavirus vaccine uptake.

This finding (or non-finding) suggests that lower rates of vaccination uptake among members of other than white ethnic groups are likely to have drivers other than or in addition to mistrust of medical, scientific, and other authorities and to negative experiences of healthcare. Experiences of healthcare explain little or no variation in uptake beyond that which is explained by trust, their association with uptake being almost entirely mediated by the latter. In some ways, this is encouraging: had the effect associated with ethnicity been found to be entirely explicable in terms of worse experiences of healthcare, then prospects of reducing it in the short term — and especially under pandemic conditions - would appear very bleak. However, the fact that so much variance remains unexplained is in itself a cause for concern, and highlights the urgent need for further research to test the mediating role of other variables. Possible candidate mediators involve sources of information, conspiracy beliefs about the novel coronavirus, and attitudes to vaccination in general [8]. Moreover, the finding that ethnic disparities in trust — a vital component in public health — could be so closely related to poorer reported experiences of NHS healthcare should serve as a reminder of the very real problem of ethnic disparities in healthcare experiences and outcomes, whether those disparities can be assumed to have a population-level impact on coronavirus vaccine uptake or not. 


\section{Limitations of this study}

This study relies on self-report data collected, and as such, it is dependent both on respondents' candour and on their recall. Also, while much effort has been made to produce a representative sample, it was not possible to obtain a probability sample. Moreover, as noted above, the focus on low income as a predictor (to which the analysts were committed by the pre-registration document) may have concealed the importance of high income as a predictor. Lastly, while the researchers were working with a relatively large sample, it included only 187 individuals who did not take up the invitation to be vaccinated, and only 234 members of other than white ethnic groups. Given that any effects associated with ethnicity must be disentangled from those associated with age, which in this context functions as a confound (both because age had a stronger association with uptake than ethnicity and because members of other than white ethnic groups were on average younger than members of white ethnic groups in the sample, as in the wider UK population), special caution must therefore be exercised in interpreting the results presented here. Further study of relationships such as those focused on in this study, for example looking for variables that might mediate the relationship between age and uptake, or that might explain the currently unexplained majority of the relationship between ethnicity and uptake, might therefore benefit either from an even larger sample or from deliberate oversampling of individuals who have not taken up the invitation to be vaccinated, and perhaps also of members of specific demographic groups.

\section{Technical note}

The analysis presented here was carried out using $\mathrm{R} v \mathrm{4}$.0.5 [28], with use of the following packages: psych v. 2.1.3 [29] for calculation of internal reliability of scales, Hmisc v. 4.5.0 [30] for calculation of weighted standard deviations, BBmisc v. 1.11 [31] for standardisation of numeric variables, mediation v. 4.5.0 [32] for mediation analysis, and WebPower v. 0.6 [33] for power analysis.

\section{Contributorship statement}

All four authors contributed to the design of the data collection instrument. Daniel Allington planned, preregistered, and executed the analysis presented here, and wrote this article together with Siobhan McAndrew, who has overall leadership of the project within which this research took place.

\section{Funding statement}

This research was supported by the Economic and Social Research Council, ES/V 015494/1.

\section{Ethics approval statement}

King's College London ethics committee, MRA-20/21-21420.

\section{Data sharing statement}

Data will be made available following the conclusion of the project's period of funding. 


\section{Competing interests statement}

The authors have no competing interests to report. 


\section{References}

1

MacDonald NE, SAGE Working Group on Vaccine Hesitancy. Vaccine hesitancy: Definition, scope and determinants. Vaccine 2015;33:4161-4.

2

Dubé E, Gagnon D, Nickels E, et al. Mapping vaccine hesitancy—country-specific characteristics of a global phenomenon. Vaccine 2014;32:6649-54. doi:10.1016/j.vaccine.2014.09.039

3

WHO. Ten threats to global health in 2019. World health organisation. 2019.https://www.who.int/newsroom/spotlight/ten-threats-to-global-health-in-2019

4

Sanche S, Lin Y, Xu C, et al. High contagiousness and rapid spread of Severe Acute Respiratory Syndrome Coronavirus 2. Emerging Infectious Diseases 2020;26:1470-7. doi:10.3201/eid2607.200282 5

Freeman D, Loe BS, Chadwick A, et al. COVID-19 vaccine hesitancy in the UK: The Oxford coronavirus explanations, attitudes, and narratives survey (OCEANS) II. Psychological Medicine 2020;1-5. doi: $10.1017 /$ S0033291720005188 6

Callaghan T, Moghtaderi A, Lueck JA, et al. Correlates and disparities of COVID-19 vaccine hesitancy. Social Science \& Medicine 2021;272. doi:10.1016/j.socscimed.2020.113638 7

Allington D, McAndrew S, Moxham-Hall VL, et al. Media usage predicts intention to be vaccinated against SARS-CoV-2 in the US and the UK. Vaccine 2021;39:2595-603. doi:10.1016/j.vaccine.2021.02.054 8

Allington D, McAndrew S, Moxham-Hall V, et al. Coronavirus conspiracy suspicions, general vaccine attitudes, trust, and coronavirus information source as predictors of vaccine hesitancy among UK residents during the COVID-19 pandemic. Psychological Medicine 2021;1-7. doi:10.1017/S0033291721001434 9

ONS. Updating ethnic contrasts in deaths involving the coronavirus (COVID-19), England and Wales: Deaths occurring 2 March to 28 July 2020. Office for National Statistics 2020. https://www.ons.gov.uk/peoplepopulationandcommunity/birthsdeathsandmarriages/deaths/articles/ updatingethniccontrastsindeathsinvolvingthecoronaviruscovid19englandandwales/ deathsoccurring2marchto28july2020

10

Byrne T, Patel P, Shrotri M, et al. Trends, patterns and psychological influences on COVID-19 vaccination intention: Findings from a large prospective community cohort study in england and wales (virus watch). medRxiv Published Online First: 2021. doi:10.1101/2021.03.22.21254130

11

Office for National Statistics. Coronavirus and vaccine hesitancy, Great Britain: 13 january to 7 february 2021. 2021.https://www.ons.gov.uk/peoplepopulationandcommunity/healthandsocialcare/

healthandwellbeing/bulletins/coronavirusandvaccinehesitancygreatbritain/13januaryto7february2021 
O’Dowd A. Covid-19: Black people over 80 in England are half as likely as white people to have been vaccinated. BMJ 2021;372. doi:10.1136/bmj.n357

13

Elgot J. Hancock: Most Bolton Covid patients eligible for jab but haven’t had it. Guardian Published Online First: 16 May 2021.https://www.theguardian.com/world/2021/may/16/hancock-defends-india-travel-bandelay-as-covid-variant-cases-mount

14

Schaffer DeRoo S, Pudalov NJ, Fu LY. Planning for a COVID-19 vaccination program. JAMA 2020;323:2458-9. doi:10.1001/jama.2020.8711

15

Mills E, Jadad AR, Ross C, et al. Systematic review of qualitative studies exploring parental beliefs and attitudes toward childhood vaccination identifies common barriers to vaccination. Journal of Clinical Epidemiology 2005;58:1081-8. doi:10.1016/j.jclinepi.2005.09.002

16

Quinn SC, Jamison AM, An J, et al. Measuring vaccine hesitancy, confidence, trust and flu vaccine uptake: Results of a national survey of White and African American adults. Vaccine 2019;37:1168-73.

17

Wilder-Smith AB, Qureshi K. Resurgence of measles in Europe: A systematic review on parental attitudes and beliefs of measles vaccine. Journal of Epidemiology and Global Health 2020;10:46-58.

doi:10.2991/jegh.k.191117.001

18

Hornsey MJ, Lobera J, Díaz-Catalan C. Vaccine hesitancy is strongly associated with distrust of conventional medicine, and only weakly associated with trust in alternative medicine. Social Science \& Medicine 2020;255. doi:10.1016/j.socscimed.2020.113019

19

Stoop N, Hirvonen K, Maystadt J-F. Institutional mistrust and child vaccination coverage in Africa. BMJ Global Health 2021;6. doi:10.1136/bmjgh-2020-004595

20

Momplaisir F, Haynes N, Nkwihoreze H, et al. Understanding drivers of coronavirus disease 2019 vaccine hesitancy among blacks. Clinical Infectious Diseases 2021;in press. doi:10.1093/cid/ciab102

21

Razai MS, Osama R, McKechnie DGJ, et al. Covid-19 vaccine hesitancy among ethnic minority groups. British Medical Journal 2021;372. doi:10.1136/bmj.n513

22

Pinder RJ, Ferguson J, Møller H. Minority ethnicity patient satisfaction and experience: Results of the National Cancer Patient Experience Survey in England. BMJ Open 2016;6. doi:10.1136/bmjopen-2016$\underline{011938}$

23

Chauhan A, Walton M, Manias E, et al. The safety of health care for ethnic minority patients: A systematic review. International Journal for Equity in Health;19. doi:10.1186/s12939-020-01223-2

24

Allington D. Do institutional trust and experiences of healthcare explain demographic disparities in coronavirus vaccination uptake? 2021.https://osf.io/dfr3m 
Wellcome. Wellcome global monitor: How does the world feel about science and health? London: : Wellcome Trust 2019.

26

Scottish Government. Inpatient experience survey. 2018.https://www.gov.scot/collections/inpatientexperience-survey/

27

Government U. Population of England and Wales.

2018.https://www.ethnicity-facts-figures.service.gov.uk/uk-population-by-ethnicity/national-and-regionalpopulations/population-of-england-and-wales/latest

28

R Core Team. R: A language and environment for statistical computing. Vienna, Austria: : R Foundation for Statistical Computing 2021. https://www.R-project.org/

29

Revelle W. Psych: Procedures for psychological, psychometric, and personality research. Evanston, Illinois: : Northwestern University 2021. https://CRAN.R-project.org/package=psych 30

Charles Dupont FEHJ with contributions from, others. many. Hmisc: Harrell miscellaneous. 2021. https://CRAN.R-project.org/package=Hmisc

31

Bischl B, Lang M, Bossek J, et al. BBmisc: Miscellaneous helper functions for B. Bischl. 2017. https://CRAN.R-project.org/package=BBmisc

32

Tingley D, Yamamoto T, Hirose K, et al. mediation: R package for Causal Mediation Analysis. Journal of Statistical Software;59:1-38. doi:10.18637/jss.v059.i05

33

Zhang Z, Mai Y. WebPower: Basic and advanced statistical power analysis. 2021. https://CRAN.Rproject.org/package $=$ WebPower

1. Linear models with standardised predictor and outcome variables. 